\title{
Planning in road evacuation: classification of exogenous activities
}

\author{
F. Russo \& C. Rindone \\ Università degli Studi Mediterranea di Reggio Calabria, DIMET - \\ Dipartimento di Informatica, Matematica, Elettronica e Trasporti, Italy
}

\begin{abstract}
Transportation planning concerns ordinary and emergency conditions. Emergency planning aims to reduce risk and relative components (occurrence; vulnerability; exposure). In this paper we consider internal transportation planning for a system in emergency conditions, with specific focus on the exposure component. One of the main macro-activities to reduce exposure is evacuation. Starting from an analysis of existing studies and best practices for classifying activities during evacuation and considering some limits of such classification, this paper uses a general classification of planning activities, with their specification for evacuation planning. Each activity is analysed in relation to strategies for transportation planning and the time reference scale (strategic, tactical, operative). A classification of transport vehicles supporting evacuation is reported.
\end{abstract}

Keywords: transportation planning, evacuation planning, classification of interventions.

\section{Introduction}

Transportation planning is a set of processes that generates different products resulting from interactions among subjects involved in the processes.

The planning process is represented by [1]:

- planning dimensions, by means of which the generic product-plan is defined that can be classified on:

- area dimension, according to the area affected by the plan, generally classified as national, regional and local; 
- time dimension, according to the time for implementing the plan, generally classified as long-term or strategic, medium-term or tactical, short-term or operative;

- study-in-depth dimension, according to the detail in planning decisions, generally classified as: directional, practicable, feasible;

- pattern of interactions among subjects involved in different planning dimensions by which a product-plan is defined; this pattern concerns a dynamic for the adoption of a single plan (internal dynamic process) and a dynamic for progress among different plans (external dynamic process).

Transportation planning concerns ordinary and emergency conditions. Transportation planning in emergency conditions aims to identify activities to reduce risk. Risk can be defined in terms of three main components: occurrence of an event in terms of probability or frequency of a specific event actually happening; vulnerability, related only to the resistance of the infrastructures when the event occurs; exposure, that is an equivalent homogeneous weighted value of people, goods and infrastructures affected during and after the event. Below we consider the exposure component. The main macro-activity to reduce exposure is evacuation, which consists in reducing the number of users and goods that can experience negative effects when emergency events occur [2].

In this paper we focus on activities for evacuation planning, starting from the macro set of general activities (material infrastructure, immaterial, equipment, management, governance and institutional). Evacuation is a complex process that depends on numerous factors. A generic evacuation plan is characterised by different elements.

The aim of this paper is to propose a classification of activities for evacuation planning. Such activities depend on events that generate emergencies. Each activity is addressed in relation to the time reference scale (strategic, tactical, operative).

The paper is organized as follows. Section 2 reports the international classification of emergency planning. In section 3 we present the proposed classification of exogenous activities for evacuation planning. In section 4, referring to equipment, a classification of vehicles for evacuation is proposed.

\section{International classifications of activities in emergency planning}

As regards the various studies in evacuation planning, an overview of methods and models to develop the evacuation planning process is presented in Goldblatt [3]. After recent events, including the 9/11 terrorist attacks and natural disasters such as hurricanes, the USA has achieved major advances in research and in applications relative to emergency planning. Transportation planning has since assumed a major role in emergency planning, management and operation $([4,5])$. The Federal Government requires all States to have a comprehensive emergency operations plan. Recommendations and guidance for evacuation planning are indicated by the US Department of Homeland Security (DHS). The US Federal 
Highway Administration (FHWA) has published a guide on evacuation planning and coordination, indicating activities to evacuate populations using highways and roads when there is advance notice of an event. American public safety organizations have worked to improve their capabilities according to the National Response Plan (NRP) and the National Incident Management System (NIMS), issued by the DHS [6].

In Australia, emergency management is defined as a range of measures to manage risks to communities and the environment. Principal measures concern different activities: risk assessment, legislation, community development, emergency response, urban development and land use management, and community recovery. The aim of emergency management is to create safe, sustainable and resilient communities that can avoid or minimise the effects of emergencies and, at the same time, have the ability to recover quickly and restore their socioeconomic vitality after an emergency event [7].

Common approaches (Australia [7], USA [8] and EU [9]) classify actions for emergency management in key functions that can be grouped in the following categories:

- $\quad$ revention/mitigation, comprising activities carried out in advance of an emergency event, with a view to reducing or eliminating its impact on the community and the environment (e.g. land management practice and planning; compliance with standards and building codes, public information campaigns and educational programs);

- $\quad$ preparedness, comprising activities to ensure, if an emergency occurs, that communities, resources and services are capable of responding to the effects (e.g. evacuation planning; exercising, training and testing of emergency service staff);

- response, including activities to control, limit or modify the emergency and to reduce its consequences (e.g. implementation of emergency plans and procedures, mobilisation of resources in response to emergency incidents and provision of immediate medical assistance);

- recovery (community), comprising the results of activities and services to support reconstruction of physical infrastructure and to manage preparedness after emergency situations (e.g. restoration of essential services, temporary housing; long-term medical care).

Activities for evacuation planning can be classified with respect to the target users into the following categories ([10-12]):

- residents within the evacuation area;

- $\quad$ systematic non-residents, comprising those who systematically reach the evacuation area for work (employees), especially school staff and pupils;

- occasional non-residents, including those who occasionally travel to the evacuation area for shopping or other activities (occasional users);

- weak users (special needs).

Evacuation plans have to determine the distribution of evacuees in the safe areas, deciding when, where, on which transportation mode and by which path 
each evacuee should travel [13]. To expedite the movement of evacuees by road, a traffic control and management strategy can be developed. To implement this strategy a set of resources is required (people with specific capabilities; equipment to assist these people, for instance traffic barriers, cones, signs; a plan that defines all the necessary details to perform evacuation operations) [14]. Evacuation operations are effective if the transport network allows rapid routing of evacuees to the safety zone [15].

As we have briefly reported, international approaches classify activities by the time sequence of the event. However, it should be pointed out that the time of preparedness (execution) differs from the time of use (implementation). While there are some activities that are used in the latter phase (e.g. temporary housing) they need to be prepared in the first phase. Hence we need a different classification that considers the time sequence of activities being carried out. Starting from this consideration we would like to propose a classification linked to execution times.

\section{Proposed classification of activities}

\subsection{General definitions}

Activities for evacuation planning can be classified into homogeneous classes in terms of execution time and the decision-maker of single activities [16]:

- activities related to material infrastructures that include interventions to modify the physical characteristics of transport system elements (e.g. geometrical characteristics of node and link infrastructures); such types of interventions can be:

o linear, if they refer to transport network links (e.g. use of an urban transportation sub-network only for emergency vehicles);

o surface (and/or nodal), if they refer to refuge areas that can be reserved for assembly points during an evacuation (e.g. areas for emergency vehicles, refuge areas);

- non-material activities that comprise consolidated research, learning and training, and others concerning telematics or Intelligent Transportation Systems;

- activities related to equipment which include interventions to provide operative tools that contribute to set up specific services in evacuation conditions; this class includes interventions to increase resource availability to support an evacuation (e.g. emergency vehicles);

- management activities comprising interventions to manage the transportation system; this class includes traffic regulations (e.g. contra flow measures, demand management, ...);

- governance and institutional activities, including interventions to regulate the transportation system; this class includes interventions to change the provisional and institutional structure regulating the mobility system; 
- Implementation of one or a set of activities can be considered a result of a rational decision-making process and has different time scales [17]:

- long-term or strategic, referring to interventions that modify the structure of the transportation system; they require long times and considerable financial resources (e.g. creation of refuge areas, modification of transport supply network) and entail changes to managerial and normative elements and to non-material infrastructures in the transport system; they affect the emission, distribution, mode and path choice components of mobility and socioeconomic characteristics (land use);

- medium-term or tactical, referring to interventions that do not modify the structure of the transportation system but produce an optimal allocation of available resources assuming minor changes (or none) in infrastructures (e.g., exercises and real experimentation); they require medium quantity of times and financial resources, and entail changes to managerial and normative elements, and to non-material infrastructures of the transport system; they affect mode and path choice components of mobility;

- short-term or operative, referring to interventions involving particular aspects of mode operations (e.g. routing of emergency vehicles in evacuation conditions); they require low quantity of time and financial resources, and entail changes to managerial and normative elements of the transport system; they affect path and mode choice components of mobility.

It can be noted that the implementation of interventions is driven by the desire to obtain results in:

- the short-medium term, including internal effects on the transport system, such as changes in the modal components of mobility (outcomes);

- the long-term, including external effects on the transport system, such as reduction in exposure to risk (goals).

\subsection{Material infrastructure activities}

In evacuation planning, material activities contribute to reducing risk within an area. Improving the infrastructural characteristics of the transport system elements can obviously enhance transport network performance: for instance, the construction of new roads can improve road network performance in evacuation conditions.

This class comprises interventions that modify the urban structure of a city. For instance, Caldaretti and Sarlo [18] propose a method to specify a Minimum Urban Structure that represents an urban sub-system including strategic public buildings, public spaces and an infrastructural network to ensure urban centre functionality in emergency conditions. The method identifies elements and interventions to reduce exposure and vulnerability components. To reduce exposure, a Minimum Urban Structure ensures transport network functionality in evacuation conditions.

Civil protection guidelines in Italy for urban emergency planning recommend identification of escape routes to allow the evacuated population to reach 
planned safe areas (emergency areas). Emergency areas are classified into: waiting areas, that allow evacuees to be assured of primary assistance after a calamitous event for a short time (e.g. parking areas, squares, public areas, ...); welcome areas, that allow evacuees to be assured of medium-long term assistance (e.g. existing buildings, hotels, tent city, ...); assembly areas that ensure assistance for rescuers (police forces, volunteers, ...). In the guidelines criteria are indicated to design escape paths and emergency areas [19].

Material infrastructure activities can be designed to increase network capacity in evacuation conditions. Some studies propose models to simulate these activities, that are formulated like a network design problem which determines where the capacity should be increased (that is on which network links) so as to achieve some system-wide objective subject to budget constraints on costs and personnel [15].

In the US emergency planning approach, building capacity is one of the principal activities of Preparedness and Response strategies, addressing the challenge to improve the transport network [6].

\subsection{Non-material activities}

In evacuation planning, non-material strategies contribute to reducing risk within an area by implementing activities:

- to increase stakeholders' knowledge in terms of research, learning and training (driver training); for instance, such improvements can contribute to modifying user behaviour in travel choices during an evacuation;

- to improve the technological characteristics of transport system elements, adopting Intelligent Transportation Systems (ITS); for instance, ITS contribute to promoting the exchange of information among stakeholders involved and supporting transport system management in emergency conditions. Such systems enhance transport system performance without modifying material infrastructures. With the use of telematic tools, different modes of transport can be connected more closely, so as to take advantage of sustainable transportation modes. ITS also work with all the other interventions, making it possible to maximise the benefits of each both individually and by integrating different classes of activities.

To increase stakeholders' knowledge in the evacuation planning process, in some countries, activities to improve the cycle between planning, training and exercises are adopted. For instance, training and exercises are key elements in evacuation planning in the United States. They include provisions for planning preparation, exercises and/or drills, and after-action reports as part of the planning process [8].

For instance, exercise programs are introduced to identify and to implement best practices. In particular, the US Department of Homeland Security (DHS) has developed the Homeland Security Exercise and Evaluation Program (HSEEP) to introduce standards for emergency exercises in terms of 
terminology, common processes and implementation approaches [20]. DHS identifies seven types of exercises with increasing levels of complexity: discussion-based, that include seminars, workshops, tabletops, games; operations-based, that include drills, functional exercises, and full-scale exercises. The HSEEP provides tools and resources to establish self-sustaining exercise programs. In this context, the US DOT includes preparedness strategies to provide, for instance, security training for transportation professionals, continuously updating the training to reflect advances in the state-of-the-art and state-of-the-practice and to meet changing training needs [8]. To increase general awareness, several agencies carry out specific activities during the year to disseminate information for hurricane preparedness [5].

ITS contribute to communicating information to evacuees during the course of an evacuation. In recent years, ITS have been installed in urban and hightraffic areas to better manage traffic flow and to assist incident management on a daily basis. The same systems are very useful in an evacuation, but are generally not available in rural and less populated areas [6].

During an evacuation, access to timely and accurate traffic information (flow rates and speeds, weather conditions, travel times, incidents, ...) is a critical activity of planning. ITS can be used to monitor traffic conditions and to disseminate travel information to evacuees such as shelter locations, alternative evacuation routes, congestion, incident information and services.

In this class, the US DOT includes cyber security strategies to integrate, for instance, effective information technology (IT) security programs with critical business functions and systems to protect the confidentiality, integrity and availability of mission critical information. The US DOT's evacuation managements applies ITS to improve notice and no-notice evacuation planning and execution, with an orientation that includes both decision makers and evacuees [20].

A national review of US evacuation plans and practices, with specific focus on the use of reverse flow operations and Intelligent Transportation Systems, is presented in Urbina and Wolshon [4].

\subsection{Equipment activities}

In evacuation planning, activities involving equipment include several subclasses, one of which concerns the purchase or procurement of vehicles required to support evacuation. In general, evacuation plans consider only evacuation by private cars and do not give adequate attention to evacuation by other land modes including buses and trains. Effective evacuation depends on the efficient use and coordination of equipment such as public transportation vehicles, and various emergency response resources [15].

There are different types of transportation vehicles to support evacuation operations. In this paper we propose a classification of activities connected to equipment. In the following ITS are not considered because their use is common to non-material and equipment strategies. 
In section 4 an analysis of transportation modes and vehicles is reported.

\subsection{Management activities}

In evacuation planning, management activities contribute to reduce risk within an area by implementing operations to organise evacuation. Traffic management, for instance, is needed for people to leave an evacuation zone. Such activities include interventions to facilitate evacuation, for instance adopting traffic control strategies (e.g. altering signalling, use of traffic control guides, using roadblocks and barriers), reverse lane/contra-flow (e.g. lane reversal and lane expansions), determining optimal evacuation paths out of an area at risk. Effects of these activities are generally simulated by applying transportation models. In the same activities interventions to manage traffic during re-entry after an evacuation are included ([22], [23]).

As part of the SICURO project, methods and models were developed to simulate evolution of a transport system from ordinary to emergency conditions after implementation of an evacuation plan ([24-28]). Specific management activities regard classes of users with special needs. Methods and models to simulate effects of these activities and to design transport services in evacuation conditions are available elsewhere ([29-33]).

\subsection{Governance and institutional activities}

In evacuation planning, governance and institutional strategies contribute to reduce risk within an area by implementing activities to improve rules and procedures that regulate evacuations. From a general point of view, institutional activities regard the laying down of rules and procedures, ex ante, in terms of laws, regulations, memoranda and so on; governance regards the application of rules and procedures, in itinere, during an evacuation.

No single institutional entity possesses expertise to act alone on the many complex issues associated with emergencies. Coordinating planning and assigning responsibilities across and among various levels of government is an evolving process [34].

Institutional activities include, for instance, policies by which political organs indicate: responsibilities of affected organizations; organization of resources and programmes; missions, priorities, cultures and performance metrics. Other activities include interagency coordination and integration relative to planning and preparation for incidents, procedures that guide incident response and management, and the informal work practices that define the way work is carried out [35].

\section{Transportation vehicles for evacuation}

Transportation vehicles for evacuation have to cover all emergency phases (prevention, preparedness, response, recovery). Here we consider transportation modes supporting evacuation procedures and specify modes in relation to users 
to evacuate (resident, systematic non-resident, occasional non-resident special needs). User categories result from an analysis of mobility demand in evacuation conditions. This analysis allows the decision maker to allocate each user category to transportation vehicles in an optimal way.

Vehicles are classified according to transportation mode and availability of equipment on board:

- land vehicles including:

○ motorised vehicles without equipment on board; this class includes all land vehicles used in emergency conditions to reach places where a disaster has occurred;

o motorised vehicles with equipment on board; this class includes all vehicles of the previous class in which special equipment (mechanical, electrical and/or telematics) are permanently installed on board; this equipment must support emergency operations; in this class other than all-purpose vehicles, we consider those for users:

- with special needs;

- with low mobility;

o non-motorised vehicles with (or without) equipment on board; this class includes all non-motorised land vehicles used to support evacuation operation during and post emergencies (kitchens, toilets, centre of operations, etc.);

- waterborne vehicles, including ships and boats;

- $\quad$ airborne vehicles comprising for instance helicopters, airplanes etc.

Land motorised vehicles without equipment on board include any kind of vehicle involved in hazardous situations. All land vehicles (cars, buses, ...) that can support evacuation operations fall into this class. A sub-classification of these vehicles concerns geometrical characteristics that have to comply with transport network characteristics (width, slope, ...). Some models are proposed to represent mobility of this vehicle class in evacuation conditions ([24-27]).

Motorised land vehicles with equipment on board include any kind of emergency vehicle equipped with specific tools devoted to tackling hazards. Emergency vehicles are essential, for instance, in evacuating those with special needs or low mobility who have no access to personal transportation. For instance, estimates of people without access to transportation in New Orleans ranged as high as $25-30 \%$ of the population. In addition to people without vehicles, potential evacuees also included the indigent, elderly, infirm, homeless, orphans, prisoners and tourists [4]. Resources to evacuate those with special needs may include staff, equipment, supplies and facilities available or potentially available for use in response [36]. The most common transportation mode for low mobility groups is the bus. In an evacuation plan that considers those with special needs or low mobility, vehicles may include school buses, public buses or emergency vehicles. Several models to design routing of services to evacuate people with special needs are proposed in the literature ([29-33]). For instance, the US DOT has defined response strategies to implement the public transit emergency management program in coordination with other DOT 
elements and other Federal agencies to ensure responsiveness to emergency transportation needs. Public transportation systems support evacuation of people that do not have vehicles and others that need assistance in evacuating. If sufficient time is available before an evacuation, public transportation systems typically may use special evacuation routes with designated pick-up points and destinations to safe areas, including shelters and other transportation transfer points [34].

Non-motorised land vehicles with (or without) equipment on board comprise any kind of transport unit used to provide services during an evacuation. This class include vehicles for dispatching provisions (e.g., medical supplies, food, etc.) [37].

\section{References}

[1] Russo F. \& Rindone C., Safety of users in road evacuation: planning internal processes and guidelines. Proc. of Urban Transport XIII, Urban Transport and the Environment in the 21st century, Brebbia C. A. (ed.), WIT Press, Southampton, pp. 825-834, 2007.

[2] Russo F. \& Vitetta A., Risk evaluation in a transportation system. International Journal of Sustainable Development and Planning, 1 (2), pp. 170-191, 2006.

[3] Goldblatt, R., Evacuation planning. Human factor and traffic engineering perspectives. Proceedings of the European Transport Conference, Strasbourg, 2004.

[4] Urbina, E., Wolshon B., National review of hurricane evacuation plans and policies: a comparison and contrast of state practices. Transportation Research Part A 37, pp. 257-275, 2003.

[5] Wolshon, B., Urbina E., Wilmot, C., Levitan, M., Review of policies and practices for hurricane evacuation. I: transportation planning, preparedness, and response. Natural Hazards Review, ASCE, pp. 129-142, 2005.

[6] Houston, N., Routes to effective evacuation planning primer series: using highways during notice evacuations operations. U. S. Department Of Transportation, Federal Highway Administration, http://ops.fhwa.dot.gov/ publications/ evac_primer / 00_evac_primer.htm (January, 2011), 2006.

[7] Australia Governments, Emergency Management Approaches, http://www.ema.gov.au/ (January, 2011), 2010.

[8] U.S. Department of Transportation, U.S. Department of Homeland Security Report to congress on catastrophic hurricane evacuation plan evaluation. www.fhwa.dot.gov/reports/hurricanevacuation/ (January, 2011), 2006.

[9] European Commission, European civil protection, http:// ec.europa.eu/ echo/ civil protection/ civil/ index.htm (January, 2011), 2011.

[10] Huang, H. C., Lo, S. M., Lin, P., Zhao, C. M., A preliminary discussion on multi-objective decision-making model for evacuation planning, Proc. of Fifth Annual IIASA-DPRI Meeting Integrated Disaster Risk Management: Innovations in Science and Policy IAEC, Beijing Normal University 14-18 September 2005. 
[11] Russo F. \& Chilà G., Safety of users in road evacuation: demand models. Proc. of Urban Transport XIII, Urban Transport and the Environment in the 21st century, Brebbia C. A. (ed.), WIT Press, Southampton, pp. 773-782, 2007.

[12] Russo F. \& Chilà G., Safety of users in road evacuation: RP vs. SP surveys in demand analysis. Proc. of Urban Transport XIV, Urban Transport and the Environment in the 21 st century, Brebbia C. A. (ed.), WIT Press, Southampton, pp. 703-713, 2008.

[13] Saadatseresht, M., Mansourian, A., Taleai, M., Evacuation planning using multiobjective evolutionary optimization approach. European Journal of Operational Research 198, pp. 305-314, 2009.

[14] Goldblatt, R., Evacuation Planning: A Key Part of Emergency Planning. Proc. of 83rd Annual Meeting Transportation Research Board January 11-15, 2004.

[15] Peeta, S., Kalafatas, G., Primary Emergency Routes for Transportation Security. Final report, Joint Transportation Research Program, FHWA/IN/JTRP-2006/1, http:// rebar.ecn.purdue.edu/ jtrp_new/ publications.aspx?id=final (January, 2011), 2008.

[16] Russo, F., Rindone, C., Safety of users in road evacuation: planning internal processes and guidelines. WIT Transactions on the Built Environment Volume 96, pp. 825-834, 2009.

[17] Russo F. \& Rindone C., Safety of users in road evacuation: the logical framework approach in evacuation planning. Proc. of Urban Transport $X I V$, Urban Transport and the Environment in the 21st century, Brebbia C. A. (ed.), WIT Press, Southampton, pp. 751-760, 2008.

[18] Caldaretti S., Sarlo A., Descrizione della struttura urbana minima e progetto urbano, in Fabietti W. (ed.), 2001.

[19] Presidenza del Consiglio dei Ministri, Manuale operativo per la predisposizione di un piano comunale o intercomunale di protezione civile. http://www.protezionecivile.it/resources/cms/documents/Manuale.pdf (March, 2011), 2007.

[20] Homeland Security Exercise and Evaluation Program (HSEEP), https://hseep.dhs.gov/pages/1001_HSEEP7.aspx (February 2009), 2007.

[21] Research and Innovative Technology Administration (RITA), U.S. Department of Transportation (US DOT), Transportation Research, Development and Technology Strategic Plan 2006-2010. http://www.rita.dot.gov/ publications/transportation_rd_t_strategic_plan/ (January, 2011), 2006.

[22] Russo F. and A. Vitetta, Reverse assignment: calibrating link cost functions and updating demand from traffic counts and time measurements, Inverse Problems in Science \& Engineering, ISSN 1741-5977 print/ISSN 1741-5985 Taylor \& Francis DOI: 10.1080/17415977.2011.565339, 2011.

[23] Sorensen, J., Vogt, B., Emergency Evacuation Guidebook http:// emc.ornl.gov /CSEPPweb/evac_files/files/body.htm (January, 2011), 2006. 
[24] Vitetta A., Musolino G. \& Marcianò F.A., Safety of users in road evacuation: Supply and demand-supply interaction models for users. Proc. of Urban Transport XIII, Urban Transport and the Environment in the 21st century, Brebbia C. A. (ed.), WIT Press, Southampton, pp. 783-792, 2007.

[25] Vitetta A., Musolino G. \& Marcianò F. A., Safety of users in road evacuation: calibration of cost functions and simulation. Proc. of Urban Transport XIV, Urban Transport and the Environment in the 21st century, Brebbia C. A. (ed.), WIT Press, Southampton, pp. 715-725, 2008.

[26] Vitetta A., Musolino G. \& Marcianò F.A., Safety of users in road evacuation: Modelling and DSS for transport supply and supply-demand interaction. Proc. of Sustainable Development and Planning IV, vol.1, Brebbia C. A. (ed.), WIT Press, Southampton, pp. 475-484, 2009.

[27] Vitetta A., Musolino G. \& Marcianò F. A., A system of models for signal setting design of a signalized road network in evacuation conditions. Proc. of Urban Transport XVI, Urban Transport and the Environment in the 21st century, Brebbia C. A. (ed.), WIT Press, Southampton, pp. 313-323, 2010.

[28] Marcianò F.A., Musolino G. \& Vitetta A., Signal setting design on a road network: application of a system of models in evacuation conditions. Proc. of Seventh International Conference on Computer Simulation in Risk Analysis and Hazard Mitigation, Brebbia C. A. (ed.), WIT Press, Southampton, 2010.

[29] Vitetta A., Quattrone A. \& Polimeni A., Safety of users in road evacuation: design of path choice models for emergency vehicles. Proc. of Urban Transport XIII, Urban Transport and the Environment in the 21st century, Brebbia C. A. (ed.), WIT Press, Southampton, pp. 803-812, 2007.

[30] Vitetta A., Quattrone A. \& Polimeni A., Safety of users in road evacuation: algorithms for path design of emergency vehicles, Proc. of Urban Transport XIV, Urban Transport and the Environment in the 21st century, Brebbia C. A. (ed.), WIT Press, Southampton, pp. 727-737, 2008.

[31] Vitetta A., Quattrone A. \& Polimeni A., Safety of users in road evacuation: Modelling and DSS for paths design of emergency vehicles, Proc. of Sustainable Development and Planning IV, vol.1, Brebbia C. A. (ed.), WIT Press, Southampton, pp. 485-495, 2009.

[32] Polimeni A., Quattrone A. \& Vitetta A., A tool for tracing emergency vehicles during evacuation, Proc. of Urban Transport XVI, Urban Transport and the Environment in the 21st century, Brebbia C. A. (ed.), WIT Press, Southampton, pp. 325-333, 2010

[33] Polimeni A., Quattrone A. \& Vitetta A., An approach to design vehicle routes in evacuation conditions, Proc. of Seventh International Conference on Computer Simulation in Risk Analysis and Hazard Mitigation, Brebbia C. A. (ed.), WIT Press, Southampton, 2010.

[34] Balog J. N., Boyd A., Caton J., Bromley P., Beth N., Strongin J., Chia D., Bagdonas K., Public Transportation Emergency Mobilization and Emergency Operations Guide, Transportation Research Board, http://onlinepubs.trb.org/onlinepubs/tcrp/tcrp_rpt_86v7.pdf (February, 2011), 2005. 
[35] Baird, M., E., Shepherd K., E., Abkowitz, M., Wegmann, F., Transportation and emergency services: identifying critical interfaces, obstacles, and opportunities. Freeway Operations and High-Occupancy Vehicle Systems 2006, Transportation Research Record: Journal of the Transportation Research Board pp 55-64, 2006.

[36] Edson, R., John, L., Webb, P., A Systems Analysis of Special-Needs Population Evacuation during Catastrophic Events. ASysT Institute, http://www.asysti.org/OurModules/SharedDocs/DocumentView.aspx? ID=113 (January, 2011), 2007.

[37] Yi W., Ozdamar L. A dynamic logistics coordination model for evacuation and support in disaster response activities, European Journal of Operational Research 179, 2007. 